\title{
Role of Filopodia in HSV-1 Entry into Zebrafish 3-O-Sulfotransferase-3- Expressing Cells
}

\author{
Samiksha Choudhary ${ }^{1}$, Lorrie Burnham ${ }^{2}$, Jeffrey M. Thompson ${ }^{3}$, Deepak Shukla ${ }^{4}$ and \\ Vaibhav Tiwari ${ }^{*}, 5$ \\ ${ }^{I}$ Department of Basic Medical Sciences, Western University of Health Sciences, Pomona, CA 91766, USA \\ ${ }^{2}$ San Bernardino Valley College, San Bernardino, CA 92410, USA \\ ${ }^{3}$ California State University, San Bernardino, CA 92407, USA \\ ${ }^{4}$ Departments of Ophthalmology and Visual Sciences \& Microbiology/Immunology, University of Illinois at Chicago IL \\ 60612, USA \\ ${ }^{5}$ Department of Microbiology \& Immunology, Midwestern University, Downers Grove, IL 60515, USA
}

\begin{abstract}
Background: Heparan sulfate proteoglycans (HSPGs) modified by zebrafish (ZF) encoded glucosaminyl 3-O sulfotransferase-3 (3-OST-3) generate a receptor for herpes simplex virus type-1 (HSV-1) entry and spread. In order to elucidate the mechanism by which HSV-1 enters into ZF-3-OST-3 cells, we investigated the mode of viral entry.

Results: Under high resolution scanning electron microscopy (SEM), actin cytoskeleton changes were observed by a dramatic increase in the number of filopodia formed during early interactions of HSV-1 with the target cells. While the increase in number was common among all the infected cells, the highest numbers of filopodia was observed in cells expressing the 3-OST-3 modified form of heparan sulfate (HS) encoded either by human or ZF. The levels of viral infection and filopodia induction were reduced with the actin polymerization inhibitors, Cytochalasin-D and Lantriculin B, suggesting an important role for actin reorganization during ZF-3-OST-3 mediated HSV-1 entry. Supporting an interesting possibility of filopodia usage during HSV-1 spread, pre-treatment of cytochalasin D in ZF-3-OST-3 cells drastically reduced virus glycoprotein induced cell fusion.

Conclusions: Taken together, our results provide new evidence on the involvement of filopodia during HSV-1 infection of ZF-3-OST-3 cells and confirm a role for modified heparan sulfate in cytoskeleton rearrangement during HSV-1 entry.
\end{abstract}

Keywords: Zebrafish, heparan sulfate, herpes simplex virus type-1,3-O- sulfotransferase-3, virus-cell interaction.

\section{BACKGROUND}

Heparan sulfate (HS) is a glycosaminoglycan (GAG) of repeating disaccharide units that consist of $\mathrm{N}$-acetylglucosamine and glucuronic/iduoronic acid $[1,2]$. They are expressed on the cell surface and in the extracellular matrix as a hybrid molecule of heparan sulfate proteoglycans (HSPGs) attached to a protein core which mediate crucial functions ranging from cell adhesion, cell signaling, blood coagulation, to wound healing and growth-factor mediated proliferation [3-5]. The long chains of HS containing alternating disaccharides are modified in a complex series of steps during its synthesis. In each of the modification steps only part of the substrate is modified resulting in high sequence diversity, which is thought to give HSPGs their functional specificity and versatility. Thus, each 3-OST can potentially generate unique protein-binding sites within HS chain $[1,6]$.

*Address correspondence to this author at the Department of Microbiology \& Immunology, Midwestern University, Downers Grove IL 60515, USA; Fax: + 1 630-515-6358; E-mail: vtiwar@midwestern.edu
The current model of herpes simplex virus type-1 (HSV1) entry suggests multiple roles for both HS and modified HS during entry and spread [7-9]. For instance, the unmodified form of HS mediates the initial viral binding or attachment to the host cells via HSV-1 glycoprotein B (gB) [10] followed by the fusion between viral envelope glycoprotein $\mathrm{D}(\mathrm{gD})$ and the host cell membrane during viral spread [11]. In the latter process of fusion it is the 3-Osulfotransferase enzymatic modification of HS chains that are involved [11-15]. Emerging evidence further suggests that HS also assists HSV-1 surfing on filopodia to reach cell body for successful infection [16].

3-OST enzymes that modify HS play an essential role during zebrafish (ZF) embryo development [17-20]. The expression patterns of multiple isoforms of 3-OST and their significance were reported in ZF [21]. Recently, we reported that the ZF encoded 3-OST-3 isoform allows HSV-1 entry similar to the human 3-OST-3 isoform [22]. However, the mechanism by which HSV-1 entry in ZF encoded 3-OST-3 cells is facilitated remains to be defined. Therefore, in this study, we examined the mechanism of HSV-1 entry via ZF encoded 3-OST-3 isoform. The results presented below 
demonstrate that HSV-1 entry into ZF encoded 3-OST-3 receptor cells is facilitated by filopodia. The significance of the actin cytoskeleton in the ZF 3-OST-3 isoform is likely to open exciting new areas of investigations. This information will be important for developing a clear understanding of HSV-1 invasion of the neurons in the established ZF model of HSV-1 infection [23]. Likewise, studying the role of 3OST-3 modified HS in ZF infection model may also contribute to our understanding of inflammation and associated pathology [24-26]. ZF has already been recognized for its value as a model for infectious diseases, inflammation, and immunity [27].

\section{RESULTS}

\section{CHO-K1 Cells Expressing ZF 3-OST-3 Isoform Induce Membrane Protrusions During HSV-1 Entry}

The previous studies have shown that primary cultures of corneal fibroblasts (CF) expressing the human encoded form of 3-OST-3 receptor leads to a change in the actin cytoskeleton during HSV-1 entry [30, 31]. Therefore, we examined whether cytoskeletal changes played any significant role in HSV-1 entry into CHO-K1 cells expressing ZF encoded 3-OST-3 isoform. To address this, a high resolution scanning electron microscopy (SEM) was performed. Wild-type HSV-1 (KOS) was added to the cells plated at a low population density for $30 \mathrm{~min}$ at $37^{\circ} \mathrm{C}$. SEM images demonstrated that the infected cells expressing $\mathrm{ZF}$ encoded 3-OST-3 had numerous virions attached to the cell body while many others were also attached to filopodia-like projections present on the plasma membrane of ZF-3-OST-3 cell (Fig. 1A, panel c). Similar projections were also noticed in human 3-OST-3 isoform expressing CHO-K1 cells (Fig. 1A, panel b). CHO-K1 cells without 3-OST-s expression showed little filopodia induction (Fig. 1A, panel a). The quantification on the number of filopodia formed in ZF-3OST-3 cells was found to be comparable to the human 3OST-3 expressing CHO-K1 cells (Fig. 1B). The above data indicates a role of ZF 3-OST-3 in filopodia induction during HSV-1 entry.

\section{Enhanced Filopodia Induction is Seen in the Cells Expressing ZF Encoded 3-OST-3 Isoform}

Because HSV-1 was able to induce filopodia both in wild-type $\mathrm{CHO}-\mathrm{K} 1$ cells and $\mathrm{CHO}-\mathrm{K} 1$ cells expressing ZF 3OST-3, we next evaluated whether changes in actin cytoskeleton were dependent on HS. In order to generate direct and visual evidence of filopodia induction during HSV-1 entry we used SEM. In this experiment HSV-1 (KOS) was used to infect pCDNA3.1 expressing CHO-K1 cells and ZF-3-OST-3 expressing CHO-K1 cells and the above infections was compared for filopodia induction to $\mathrm{CHO}$ cells that are deficient in $\mathrm{HS}$ (CHO-745). As shown in Fig. (2A, panel a) HSV-1 infected CHO-745 (HS deficient) cells failed to produce significant amount of filopodia compared to the HS expressing wild-type CHO-K1 cells (Fig $\mathbf{2 A}$, panel b). In parallel, pronounced filopodia were observed in CHO-K1 cells expressing ZF encoded 3-OST-3 isoform (Fig. 2A, panel c). Quantification of percentage of cells forming filopodia was highest in ZF-3-OST-3 cells (Fig. 2B). These results indicate that filopodia induction during HSV-1 entry is HS dependent; Furthermore, the presence of 3-OST-3-modified receptor significantly enhances filopodia induction to facilitate HSV-1 entry into ZF expressing 3-OST-3 CHO-K1 cells.

\section{Role for Actin Cytoskeleton During HSV-1 Entry into Cells Expressing ZF 3-OST-3}

To further demonstrate the significance of actin network in HSV-1 entry, both ZF and human 3-OST-3 receptor expressing CHO-K1 cells were pre-treated with inhibitors of actin polymerization such as cytochalasin D (Cyto D) and lantriculin B (Lat B) [32-34] or mock treated before infecting with Lac $Z$ encoded $\beta$-galactosidase expressing HSV-1. It was postulated that pre-treatment would have negative effect on entry provided the actin-based protrusions (such as filopodia) played a role in the virus attachment as well as entry in to cells. As shown in Fig. $(\mathbf{3 A}, \mathbf{B})$, both of the actin depolymerizers blocked HSV-1 entry in a dosage dependent manner. To generate further visual evidence, SEM was performed in HSV-1 infected ZF-3-OST-3 expressing CHO-K1 cells in presence and absence of Cyto D. Corresponding effects on the inhibition on the number of filopodia and HSV-1 entry was noticed when cells were preincubated with Cyto-D (Fig. 3C, D). The quantitation of the above-mentioned data suggests that significant changes in the cytoskeleton occur during initial phase of HSV-1 infection in ZF encoded 3-OST-3 receptor. A similar increase in cellular projections was previously reported with other HSV-1 receptors including nectin-1 [35].

\section{ZF Encoded 3-OST-3 Mediated HSV-1 Glycoprotein Induced Cell-to-Cell Fusion is Actin Cytoskeleton Dependent}

After establishing the role of ZF encoded 3-OST-3 in filopodia induction during HSV-1 entry, we next examined whether ZF 3-OST-3 mediated cell-to-cell fusion is also an actin network dependent process. CHO-K1 cells were used that are resistant to virus-induced cell fusion due to the absence of a $\mathrm{gD}$ receptor [28]. A previously described luciferase reporter gene assay was performed to quantify the induced cell fusion between 3-OS HS cells modified by ZF encoded 3-OST-3 and HSV-1 glycoproteins in presence and absence of Cyto D [28]. The '"effector' CHO-K1 cells were transiently transfected with each of four glycoprotein plasmids: pPEP98 (gB), pPEP99 (gD) pPEP100 (gH), and pPEP101 (gL), as well as the plasmid pT7EMCLuc that expresses a luciferase reporter gene [29]. The "target" cells were transfected with a 3-OST plasmid expressing ZF encoded 3-OST-3 and the plasmid pCAGT7, which expresses T7 RNA polymerase to induce expression of the luciferase gene. For a negative control, cells were transfected with T7 RNA polymerase and control vector pCDNA3.1. The cells expressing human 3-OST-3 and T7RNA polymerase served as a positive control. As shown in Fig. (4) a slightly higher amount of fusion occurred in human 3-OST-3 isoform, while ZF encoded 3-OST-3 expressing cells also showed fusion (grey bar) compared to negative control cells without 3-OST-3 expression (black bar). Interestingly significant inhibition in cell-to-cell fusion was observed in presence of Cyto D (grey bars with black lines). These results reinforce our finding that changes in actin cytoskeleton play a critical role during HSV-1 entry and spread and cells expressing ZF 3-OST-3 facilitate filopodia induction. 

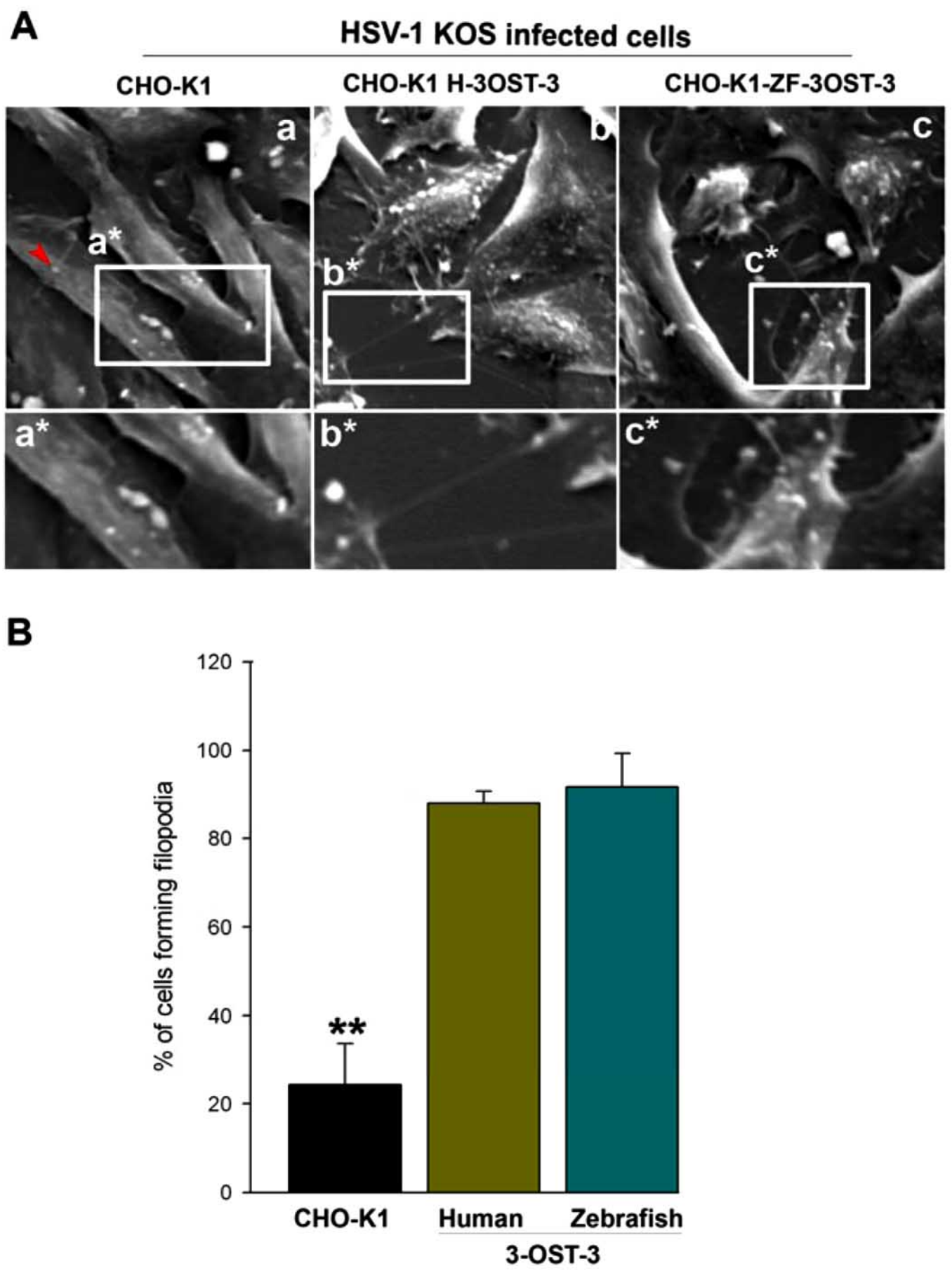

Fig. (1). Role of filopodia during HSV-1 entry into Chinese hamster ovary (CHO-K1) cells expressing Zebrafish (ZF) encoded 3OST-3 receptor. (A) Scanning electron microscopy (SEM) performed on HSV-1 (KOS) infected glycoprotein D (gD) receptor negative (wild-type CHO-K1 cells; panel a) and gD-receptor positive cells (panel b and panel c). CHO-K1 cells expressing human 3-OST-3 (H-3OST-3), and zebrafish (ZF) encoded 3-OST-3 (ZF-3-OST-3) were grown at a confluence of $40 \%$ in a chamber slides (Lab-Tek chamber slide) and were exposed to HSV-1 (25 pfu/cell for $45 \mathrm{~min}$ at $37^{\circ} \mathrm{C}$ ). The infected cells were fixed with $2 \%$ formaldehyde/4\% glutaraldehyde in PBS before SEM. Highlighted regions showing virions bound to wild-type CHO-K1 cells (panel a*) were unable to induce filopodia (red arrow indicates single Filopodia in panel a), while large number of filopodia (panels $\mathrm{b}^{*}$ and $\mathrm{c}^{*}$ ) were observed in human and $\mathrm{ZF}$ encoded 3OST-3 expressing CHO-K1 cells. The arrow indicates presence of virus on the induced filopodia. (B) Determination of percentage of filopodia scored from sampled groups of 100 single cells or clusters (with 5-20 cells) in wild-type CHO-K1 cells, and CHO-K1 cells expressing human and ZF 3-OST-3 receptor in triplicate experiments, $5 \mu \mathrm{m}$ length of a protrusion and at least $10 \%$ of the cell surface covered with 25 or more protrusions is scored positive. ${ }^{* *} \mathrm{P}<0.05$, one way ANOVA. 


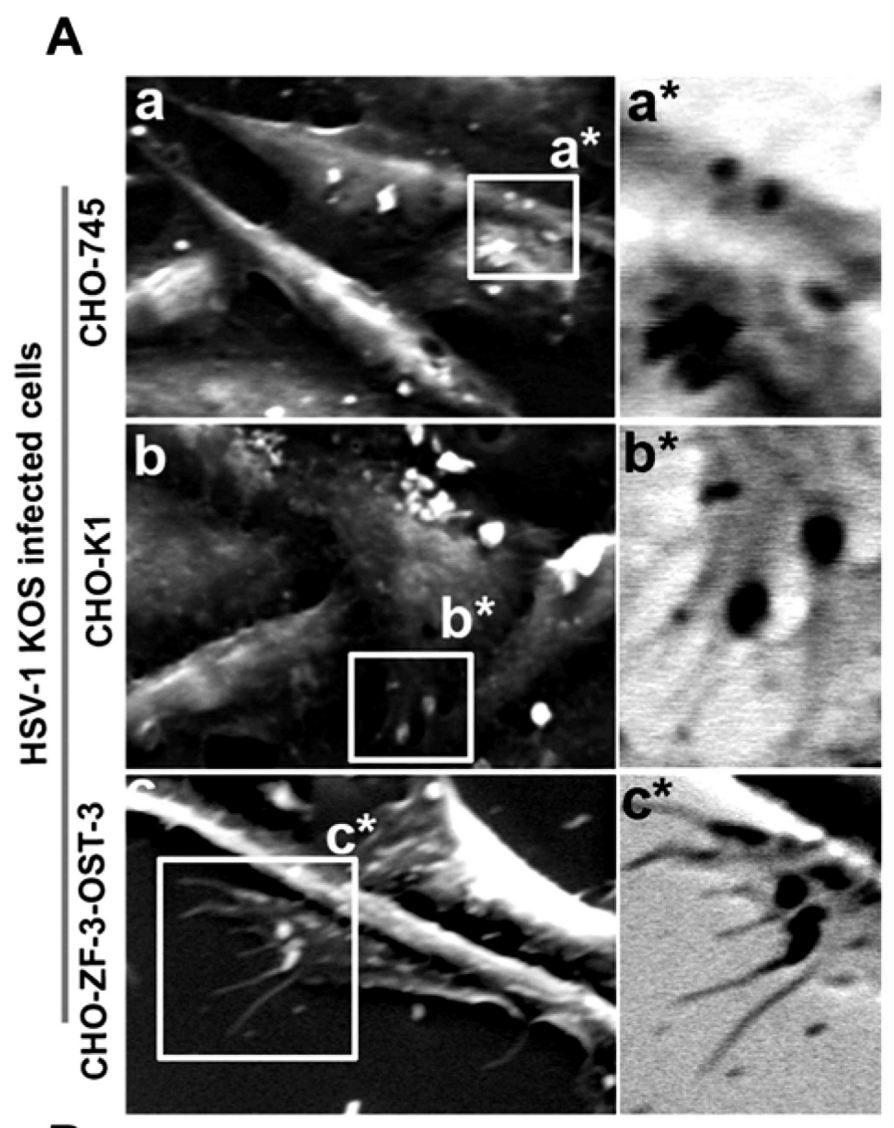

B

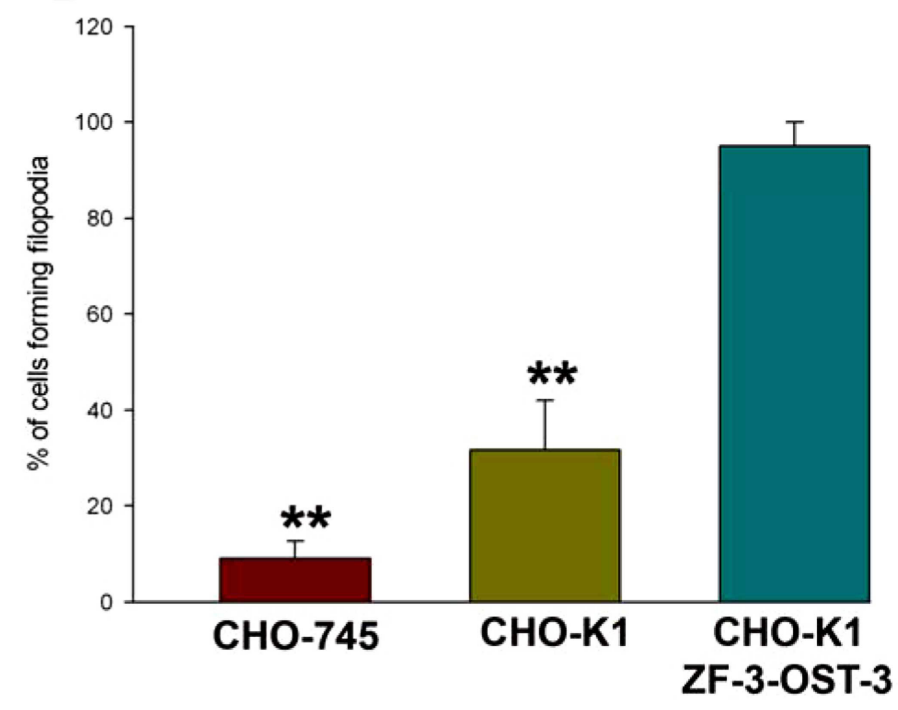

Fig. (2). HSV-1 interaction with zebrafish (ZF) encoding 3-OST-3 leads to enhanced filopodia induction. (A) Scanning electron microscopy (SEM) performed on heparan sulfate (HS) negative (CHO-745 cells; panel a) and HS positive (wild-type CHO-K1; panel b and ZF expressing 3-OST-3; panel c) cells in presence of HSV-1 (25 pfu/cell) for $45 \mathrm{~min}$ at $37^{\circ} \mathrm{C}$. The regions highlighted for filopodia in panels $\mathrm{a}, \mathrm{b}$ and $\mathrm{c}$ has been inverted as $\mathrm{a}^{*}, \mathrm{~b}^{*}$, and $\mathrm{c}^{*}$. Highlighted regions showing virions bound to CHO-745 cells (panel $\mathrm{a}^{*}$ ) were unable to induce filopodia, while small number of filopodia were observed in virions bound CHO-K1 cells (panels $b^{*}$ ). The maximum number of filopodia were observed in ZF encoded 3-OST-3 expressing CHO-K1 cells (panel $\mathrm{c}^{*}$ ). (B) Determination of percentage of filopodia scored in HSV-1 (25 pfu/cell for $45 \mathrm{~min}$ at $37^{\circ} \mathrm{C}$ ) treated CHO-745, CHO-K1 and CHO-ZF-3-OST-3 is presented. Sampled groups of 50 single cells or clusters (with 5-20 cells) in triplicate experiments, $5 \mu \mathrm{m}$ lengths of a protrusion and at least $10 \%$ of the cell surface covered with 25 or more protrusions is scored positive. $* * \mathrm{P}<0.05$, one way ANOVA. 

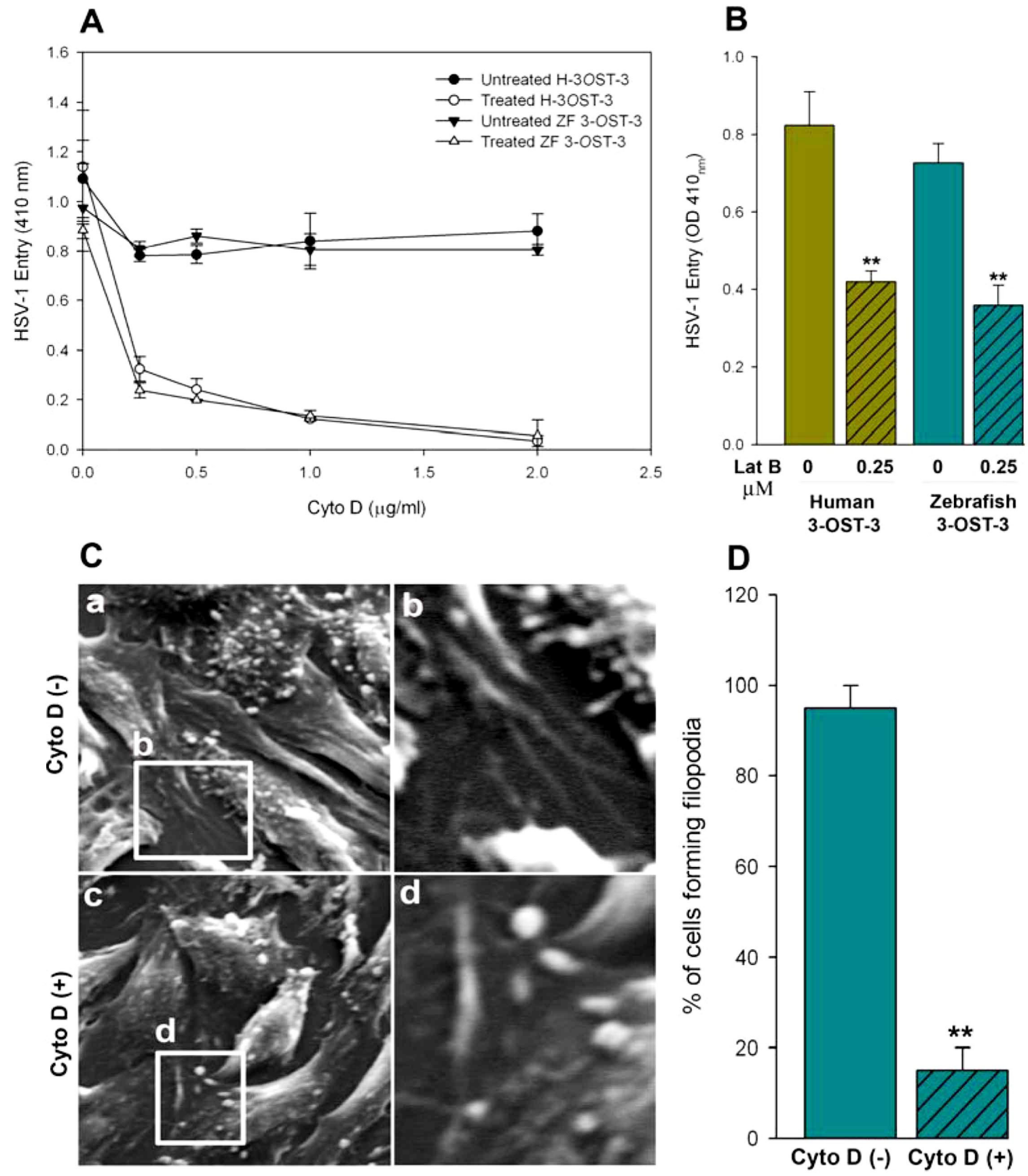

Fig. (3). Actin depolymerizers block HSV-1 entry into zebrafish (ZF) 3-OST-3 expressing CHO-K1 cells. (A, B) Cultured monolayers of cultured human $(\mathrm{H})$ and zebrafish $(\mathrm{ZF})$ encoded 3-OST-3 CHO-K1 cells were pre-treated with the indicated concentrations of the actin depolymerizing agent Cytochalisin D (Cyto D; panel A) and Lanticulin B (Lat B; panel B) before exposed to $\beta$-galactosidase expressing HSV-1 gL86 (25 pfu/cell) virus. Cells treated with $1 \times$ PBS treated cells were used as a control. Viral entry was quantitated 6 h after infection at $410 \mathrm{~nm}$ using a spectrophotometer. The experiment was repeated three times with similar results. (C) Visualization on the inhibition of filopodia via scanning electron microscopy (SEM) on HSV-1 infected ( $25 \mathrm{pfu} / \mathrm{cell}$ for 45 min at $\left.37^{\circ} \mathrm{C}\right) \mathrm{ZF}-3-O S T-3$ expressing CHO-K1 cells in absence (panel a) and presence (panel c) of Cyto D at $0.5 \mu \mathrm{g} / \mathrm{ml}$. The regions in panel a (filopodia induction) and panel c (reduced filopodia) highlighted are shown in panel b and d. (D) Determination of percentage of filopodia scored in ZF-3-OST-3 infected cells in absence and presence of Cyto D. Sampled groups of 50 single cells or clusters (with 5-20 cells) in a triplicate experiment, $5 \mu \mathrm{m}$ length of a protrusion and at least $10 \%$ of the cell surface covered with 25 or more protrusions is scored positive. $* * \mathrm{P}<0.05$, one way ANOVA. 


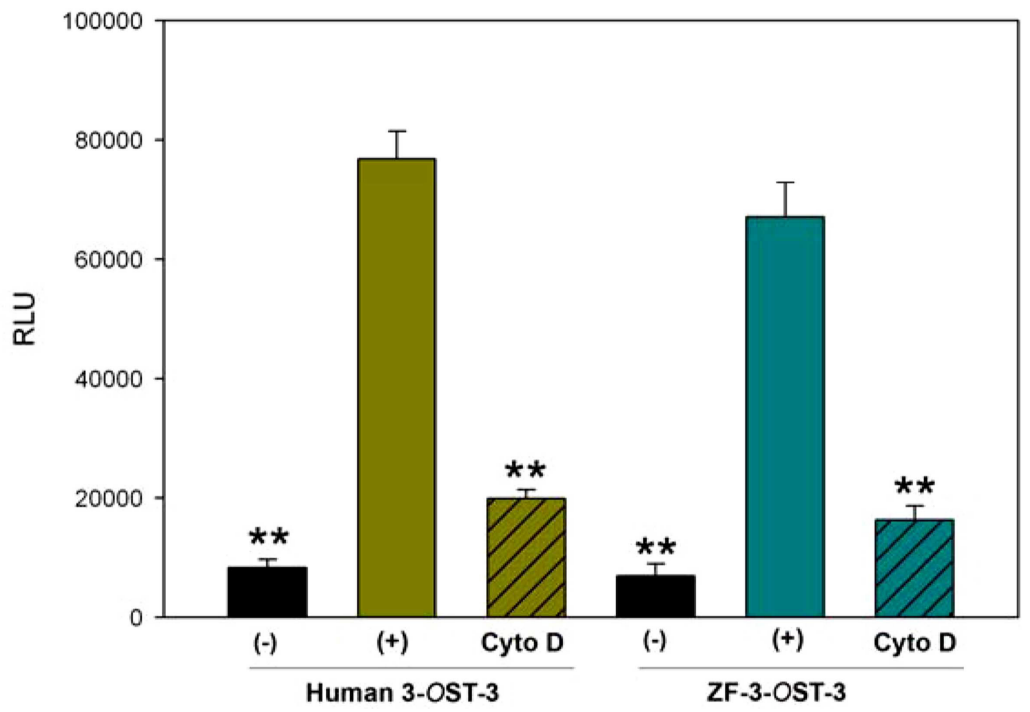

Fig. (4). Actin depolymerizer cytochalasin D negatively affects HSV-1 glycoprotein mediated cell fusion with zebrafish (ZF) encoded3-OST-3 receptor. In this experiment target CHO-K1 cells expressing either human 3-OST-3 or ZF encoded 3-OST-3 along with luciferase gene were pre-incubated with Cyto D $(0.5 \mu \mathrm{g} / \mathrm{ml})$ or with $1 \times$ PBS for $45 \mathrm{~min}$ at room temperature before co-culturing with effector cells expressing four essential HSV-1 glycoproteins ( $\mathrm{gB}, \mathrm{gD}, \mathrm{gH}-\mathrm{gL})$ or control plasmid (pCDNA3.1) along with T7 Polymerase in 1:1 ratio for $24 \mathrm{hrs}$. The graph shows the quantitative cell fusion in presence (line bar) and absence of Cyto D (plain bar). The black bar shown is a negative control. Data represent the means standard deviations of results in triplicate wells from a representative experiment. $* * \mathrm{P}<0.05$, one way ANOVA.

\section{DISCUSSION}

Our previous findings have shown the role of zebrafish (ZF) encoded 3-OST-3 as a receptor for HSV-1 gD [22]. Here we examined some additional but imoprtant aspects of HSV-1 entry mediated by ZF-3-OST-3 isoform. The novel finding presented herein is that ZF encoded 3-OST-3 receptor cells show significant changes in actin cytoskeleton during initial phase of HSV-1 entry (Fig. 1A, panel $\mathrm{c}$ and Fig. 2A, panel c). Similarly, we demonstrate a role for heparan sulfate, and more importantly modified heparan sulfate, in filopodia induction. Our data suggests a novel role for ZF encoded 3-OST-3 isoform in the induction of filopodia. It has been shown previously that HSV-1 uses filopoida for surfing to efficiently reach the cell body for entry and that surfing is required for enhanced infection [16]. Although induction of filopodia was not totally dependent on a $\mathrm{gD}$ receptor as $\mathrm{CHO}-\mathrm{K} 1$ cells expressing heparan sulfate (HS) without any 3-OSTs were able to induce filopodia (Fig. $\mathbf{2 A}$, panel b); however, a significant increase in the amount of filopodia was noticed in presence of a $\mathrm{gD}$ receptor i.e. CHO-K1 cells expressing either ZF or human form 3-OST-3 receptor. The above finding implies that ZF encoded 3-OST3 may provide unique gD binding sites for enhanced cell signaling and filopodial development. Specific structures within HS are known to guide retinal axons [36]. In addition, HS binding protein, VEGF (vascular endothelial growth factor), is also involved in filopodia formation [37]. Therefore, it is likely that actin cytoskeletal changes as a result of HSV-1 gD interaction to ZF-3-OST-3 allow not only virus-cell fusion [22] but may also enhance viral surfing to enhance viral infectivity.

The presented SEM images indicate that pronounced filopodia induction with phagoctyic arms is observed during HSV-1 entry into ZF-3-OST-3 expressing CHO-K1 cells
(Fig. 1A, panel c, and Fig. 2A, panel c). The filopodia associated arms were similar to the HSV-1 phagoctyic-like uptake by human corneal fibroblasts (CF) cells where 3OST-3 plays a major role for HSV-1 entry and spread [30]. These findings support previous studies that $\mathrm{HS}$ and 3-OS HS work at two different levels to promote HSV infection. First, it is well documented that HSV-1 glycoprotein B (gB) contact to cell surface HS to induce filopodia which aid in lateral viral movement along the length of filopodia to bring the virions closer to the cell body [16]. Second, HSV-1 glycoprotein $\mathrm{D}(\mathrm{gD})$ interaction to $3-\mathrm{OST}-3$ receptor triggers the viral uptake via filopodia [30]. The role of actin cytoskeleton is widely implicated in microbial pathogenesis $[38,39]$. Multiple viruses exploit the host actin cytoskeleton to facilitate important aspects of their lifecycles including viral surfing, entry into target cell, egress, and intercellular spread [40].

\section{CONCLUSION}

The knowledge that HSV-1 entry into ZF 3-OST-3 cells involves filopodial induction can be useful for the following reasons: 1) it can lead the way for the development of antiZF-3-OST-3 compounds that can potentially block HSV-1 entry and spread at an early step of viral infection i.e. during induction of filopodia. 2) Because the 3-OST-3 gene family is differentially regulated in ZF [21], an important question that remains to be demonstrated is whether ZF encoded 3OST-3 isoform is unique for filopodia development or other isoforms of $\mathrm{ZF}$ encoded 3-OS HS can also function as a $\mathrm{gD}$ receptor and allow filopodial induction and 3) A ZF model can be also established to study the in vivo significance of filopodia during HSV-1 infection.

In summary, apart from showing the significance of ZF encoded 3-OST-3 in filopodia induction, our study also implicates HS in filopodia induction, which is sure to pave 
the way for examining the roles of HS and modified HS in cellular signaling associated with cytoskeletal changes. Future understanding regarding HSV-1 usage of HS and 3$O S$ HS to facilitate viral entry and spread via filopodia in ZF model is likely to open up new ways to develop anti-HSV agents and strategies to prevent both viral spread and inflammation.

\section{METHODS}

\section{Plasmids}

The Zebrafish encoded 3-OST-3 gene was cloned into pDream2.1 plasmid vector (Genscript), while the Human 3OST-3 expressing plasmid (pDS43) was provided by Dr. Shukla (University of Illinois at Chicago) [11]. The HSV-1 (KOS) glycoprotein expressing plasmids used were pPEP98 (gB), pPEP99 (gD), pPEP100 (gH), and pPEP101 (gL) [28]. Other plasmids used in this study include pCAGT7 (T7 RNA polymerase), and pT7EMCLuc (luciferase gene) for the luciferase assay [29], and a control empty vector pCDNA3.1 from Invitrogen (Carlsbad, CA, USA).

\section{Cell Culture and Viruses}

Wild-type Chinese Hamster ovary-K1 (CHO-K1) and heparan sulfate defective cells (CHO-745) were kindly provided by P.G. Spear (Northwestern University, Chicago). Both CHO-K1 and CHO-745 cells were grown in Ham's F12 medium (Gibco/BRL, Carlsbad, CA) supplemented with $10 \%$ fetal bovine serum (FBS), and penicillin and streptomycin (Gibco/BRL). The $\beta$-galactosidase expressing recombinant HSV-1 (KOS) gL86 was provided by P.G. Spear (Northwestern University, Chicago).

\section{Scanning Electron Microscopy (SEM)}

Wild-type CHO-K1, CHO-745 cells and CHO-K1 cells expressing human $(\mathrm{H})$ and zebrafish $(\mathrm{ZF}) 3-O S T-3$ receptor were infected with HSV-1 (KOS) at $25 \mathrm{pfu} /$ cell for $45 \mathrm{~min}$ at $37^{\circ} \mathrm{C}$ in triplicate experiments. In a parallel experiment, the CHO-K1 cells expressing ZF-3-OST-3 were pre-incubated with $1 \times$ PBS or with an actin polymerizer cyto-D followed by HSV-1 infection at $25 \mathrm{pfu} /$ cell for $45 \mathrm{~min}$ at $37^{\circ} \mathrm{C}$. The cells were then fixed with $2 \%$ formaldehyde $/ 4 \%$ glutaraldehyde in $1 \times$ phosphate buffer saline (PBS) prior to SEM study. This was followed by fixing cells with $1 \%$ osmium tetroxide formaldehyde/glutaraldehyde for 40 minutes. Dehydration was done using 25\% ethanol, 50\% ethanol, $70 \%$ ethanol, $90 \%$ ethanol, $95 \%$ ethanol, $100 \%$ ethanol at five minutes each respectively. $100 \%$ ethanol was repeated to ensure dehydration. Cover slips were removed from dishes and mounted on aluminum studs previously cleaned with $100 \%$ ethanol. Cover slip edges were painted with colloidal silver for conduction and dried in a Critical Point Dryer (Samdri-780A). Samples were then coated with gold using a Sputter Coater (Hummer VI-A) for 2.5 minutes. Samples were viewed using a Hitachi S-2700 Scanning Electron Microscope (SEM). Images were captured at 10005000x using Revolution image capture system and a controller/analysis system for energy dispersive X-ray spectroscopy.

\section{HSV-1 Entry Assay}

As previously described [22] CHO-K1 cells were grown in 6-well plates to subconfluence and transfected with $2.5 \mu \mathrm{g}$ of human and or ZF encoded 3-OST isoforms (3-OST-3) or control plasmid (pDream2.1 or pCDNA3.1) using LipofectAMINE (Gibco/BRL) [22]. At $16 \mathrm{~h}$ posttransfection, the cells were replated into 96-well dishes for pre-treatment with actin depolymerizers (cyto-D and lant-B at indicated concentrations for $45 \mathrm{~min}$ at room temperature) followed by infection with $\beta$-galactosidase expressing recombinant HSV-1 gL86 virus. After 6 -h post infection, $\beta$ galactosidase assay were performed using a soluble substrate o-nitrophenyl- $\beta$-D-galactopyranoside (ONPG; ImmunoPure, Pierce). The enzymatic activity was measured at $410 \mathrm{~nm}$ using a micro-plate reader [22].

\section{HSV-1 Glycoprotein Induced Cell-Fusion Assay}

A cell-to-cell fusion assay described previously was used $[22,28]$. CHO-K1 cells were grown in 6-well plates to subconfluent levels. The cultured CHO-K1 "target" cells were transfected with plasmids expressing either human or zebrafish (ZF) 3-OST-3 isoform and the luciferase gene. The " "effector'" or virus-like cells were co-transfected with four HSV-1(KOS) glycoproteins as previously described [28]. In either case, the total amount of DNA used for transfection was kept constant. For transfection, $\mathrm{CHO}-\mathrm{K} 1$ cells were grown to $70 \%$ confluency in a 6 well dishes. $2.5 \mu \mathrm{g}$ of plasmid DNA was mixed with $8 \mu \mathrm{l}$ of Lipofectamine (Gibco/BRL) in to a total volume of $1 \mathrm{ml}$ with serum-free media. After $16 \mathrm{~h}$, target and effector cells were mixed in a $1: 1$ ratio and then replated in $24-w e l l$ dishes. In parallel effector cells transfected only with pCDNA3.1 along with T7 RNA polymerase was mixed with the target cells expressing either human or ZF 3-OST-3 isoform was used as a negative control. The activation of the reporter luciferase gene as a measure of cell fusion was examined after $24 \mathrm{~h}$. Luciferase activity was quantified using the luciferase reporter assay system (Promega, Madison, WI).

\section{CONFLICT OF INTEREST}

The authors confirm that this article content has no conflict of interest.

\section{ACKNOWLEDGEMENTS}

We sincerely thank Dr. Spear (Northwestern University, Chicago) for providing the reagents and the facility at California State University (San Bernardino) for electron microscopy. This work was supported by National Institutes of Health RO1 grants AI057860 (to D. S.) and R15 (1R15AI088429-01A1) to V.T.

\section{REFERENCES}

[1] Esko JD, Lindahl U. Molecular diversity of heparan sulfate. J Clin Invest 2001; 108: 169-73.

[2] Lindahl U, Kusche-Gullberg M, Kjellen L. Regulated diversity of heparan sulfate. J Biol Chem 1998; 273: 24979-82.

[3] Gorsi B, Stringer SE. Tinkering with heparan sulfate sulfation to steer development. Trends Cell Biol 2007; 17: 173-7.

[4] Esko JD, Selleck SB. Order out of chaos: assembly of ligand binding sites in heparan sulfate. Annu Rev Biochem 2002; 71: 43571.

[5] Bishop JR, Schuksz M, Esko JD. Heparan sulphate proteoglycans fine tune mammalian physiology. Nature 2007; 446: 1030-7.

[6] Shukla D, Spear PG. Herpesviruses and heparan sulfate: an intimate relationship in aid of viral entry. J Clin Invest 2001; 108: 503-10.

[7] Spear PG, Longnecker R. Herpesvirus entry an update. J Virol 2003; 77: 10179-85. 
[8] O'Donnell CD, Shukla D. The Importance of Heparan Sulfate in Herpesvirus Infection. Virol Sin 2008, 23: 383-93.

[9] O'Donnell CD, Kovacs M, Akhtar J, Valyi-Nagy T, Shukla D. Expanding the role of 3-O sulfated heparan sulfate in herpes simplex virus type-1 entry. Virology 2010; 397: 389-98.

[10] WuDunn D, Spear PG. Initial interaction of herpes simplex virus with cells is binding to heparan sulfate. J Virol 1989; 63: 52-8.

[11] Shukla D, Liu J, Blaiklock P, et al. A novel role for 3-O-sulfated heparan sulfate in herpes simplex virus 1 entry. Cell 1999; 99: 1322.

[12] O’Donnell CD, Tiwari V, Jin-Oh M, Shukla D. A Role for 3-Osulfotransferase isoform-2 in assisting HSV-1 entry and spread. Virology 2006; 346: 452-9.

[13] Tiwari V, O'Donnell CD, Oh MJ, Valyi-Nagy T, Shukla D. A role for 3-O-sulfotransferase isoform-4 in assisting HSV-1 entry and spread. Biochem Biophys Res Commun 2005. 338: 930-7.

[14] Xia G, Chen J, Tiwari V, et al. Heparan sulfate 3-Osulfotransferase isoform 5 generates both an antithrombin-binding site and an entry receptor for herpes simplex virus, type 1. J Biol Chem 2002; 277: 37912-9.

[15] Xu D, Tiwari V, Xia G, Clement C, Shukla D, Liu J. Characterization of heparan sulphate 3-O-sulphotransferase isoform 6 and its role in assisting the entry of herpes simplex virus type 1. Biochem J 2005; 385: 451-9.

[16] Oh MJ, Akhtar J, Desai P, Shukla D. A role for heparan sulfate in viral surfing. Biochem Biophys Res Commun 2010; 391: 176-81.

[17] Bink RJ, Habuchi $\mathrm{H}$, Lele $\mathrm{Z}$, et al. Heparan sulfate 6-Osulfotransferase is essential for muscle development in zebrafish. $\mathrm{J}$ Biol Chem 2003; 278: 31118-27.

[18] Chen E, Stringer SE, Rusch MA, Selleck SB, Ekker SC. A unique role for 6-O sulfation modification in zebrafish vascular development. Dev Biol 2005; 284: 364-76.

[19] Zhang F, Zhang Z, Thustle R, et al. Structural characterization of glycosaminoglycans from zebrafish in different ages. Glycoconj $\mathrm{J}$ 2009; 26: 211-8

[20] Liu IH, Zhang C, Kim MJ, Cole GJ. Retina development in zebrafish requires the heparan sulfate proteoglycan Agrin. Dev Neurobiol 2008; 68(7): 877-98.

[21] Cadwallader AB, Yost HJ. Combinatorial expression patterns of heparan sulfate sulfotransferase in zebrafish: I. The 3-Osulfotransferase family. Dev Dynam 2006; 235: 3423-31.

[22] Hubbard S, Darmani NA, Thrush GR, et al. Zebrafish-encoded 3O-sulfotransferase-3 isoform mediates herpes simplex virus type 1 entry and spread. Zebrafish 2010, 7:181-7.

[23] Burgos JS, Gomez JR, Alfaro JM, Sastre I, Valdivieso F. Zebrafish as a new model for herpes simplex virus type 1 infection. Zebrafish 2008; 5: 323-33.
[24] Parish CR. The role of heparan sulphate in inflammation. Nature Rev Immunol 2006; 6: 633-43.

[25] Iozzo RV, San Antonio JD. Heparan sulfate proteoglycans: heavy hitters in the angiogenesis arena. J Clin Invest 2001, 108: 349-55.

[26] Aquino RS, Lee ES, Park PW. Diverse functions of glycosaminoglycans in infectious diseases. Prog Mol Biol Transl Sci 2010; 93: 373-94.

[27] Sullivan C, Kim CH. Zebrafish as a model for infectious disease and immune function. Fish Shellfish Immunol 2008; 25: 341-50.

[28] Tiwari V, Clement C, Duncan MB, Chen J, Liu J, Shukla D. A role for 3-O-sulfated heparan sulfate in cell fusion induced by herpes simplex virus type 1. J Gen Virol 2004; 85: 805-9.

[29] Pertel P, Fridberg A, Parish M, Spear PG. Cell fusion induced by herpes simplex virus glycoproteins $\mathrm{gB}, \mathrm{gD}$, and $\mathrm{gH}$-gL requires a gD receptor but not necessarily heparan sulfate. Virology 2001; 279: 313-24.

[30] Clement C, Tiwari V, Scanlon PM, Vali-Nagy T, Yue BYJT, Shukla D. A novel for phagocytosis-like uptake in herpes simplex virus entry. J Cell Biol 2006; 174: 1009-21.

[31] Tiwari V, Clement C, Xu D, et al. Role for 3-O-sulfated heparan sulfate as a receptor for herpes simplex virus type- 1 entry into primary human corneal fibroblasts. J Virol 2006; 80: 8970-80.

[32] Gottlieb TA, Ivanov IE, Adesnik M, Sabatini DD. Actin microfilaments play a critical role in endocytosis at the apical but not the basolateral surface of polarized epithelial cells. J Cell Biol 1993; 120: 695-710.

[33] Rabinovitch M. Professional and non-professional phagocytes: an introduction. Trends Cell Biol 1995; 5: 85-7.

[34] Schafer DA. Regulating actin dynamics at membranes: a focus on dynamin. Traffic 2004; 5: 463-9.

[35] Tiwari V, Oh MJ, Kovacs M, Shukla SY, Valyi-Nagy T, Shukla D. Role for nectin-1 in herpes simplex virus 1 entry and spread in human retinal pigment epithelial cells. FEBS J 2008; 275: 5272-85.

[36] Ogata-Iwao M, Inatani M, Iwao $\mathrm{K}$, et al. Heparan sulfate regulates intraretinal axon pathfinding by retinal ganglion cells. Invest Ophthalmol Vis Sci 2011; 52: 6671-9.

[37] Stenzel D, Lundkvist A, Sauvaget D, et al. Integrin-dependent and -independent functions of astrocytic fibronectin in retinal angiogenesis. Development 2011; 138: 4451-63.

[38] Döhner K, Sodeik B. The role of the cytoskeleton during viral infection. Curr Top Microbiol Immunol 2005; 285: 67-108.

[39] Greber UF, Way M. A superhighway to virus infection. Cell 2006; 124: 741-54.

[40] Radtke K, Döhner K, Sodeik B. Viral interactions with the cytoskeleton: a hitchhiker's guide to the cell. Cell Microbiol 2006; 3: $387-400$. 